\title{
The impact of introducing Brayden Illuminating CPR manikins on Basic Life Support exam performance at a large University in the UK - a randomised controlled trial
}

\section{Introduction:}

Early bystander cardiopulmonary resuscitation (CPR) improves outcomes after cardiac arrest $(1,2)$. Bystander CPR performance can be encouraged by offering training to potential lay rescuers. (3) Brayden's Illuminating CPR Manikin offers a new approach to training with real-time feedback. The primary objective of this study was to ascertain if candidates' chest compression and 'hands off time' (time without chest compressions) performance varies according to type of training manikin used.

\section{Materials and Methods:}

Following a pilot/feasibility phase to estimate sample size, this randomised controlled trial was conducted as part of the University of Birmingham's European Resuscitation Council (ERC) Basic Life Support (BLS) provider courses between October 2017 and April 2018 (see

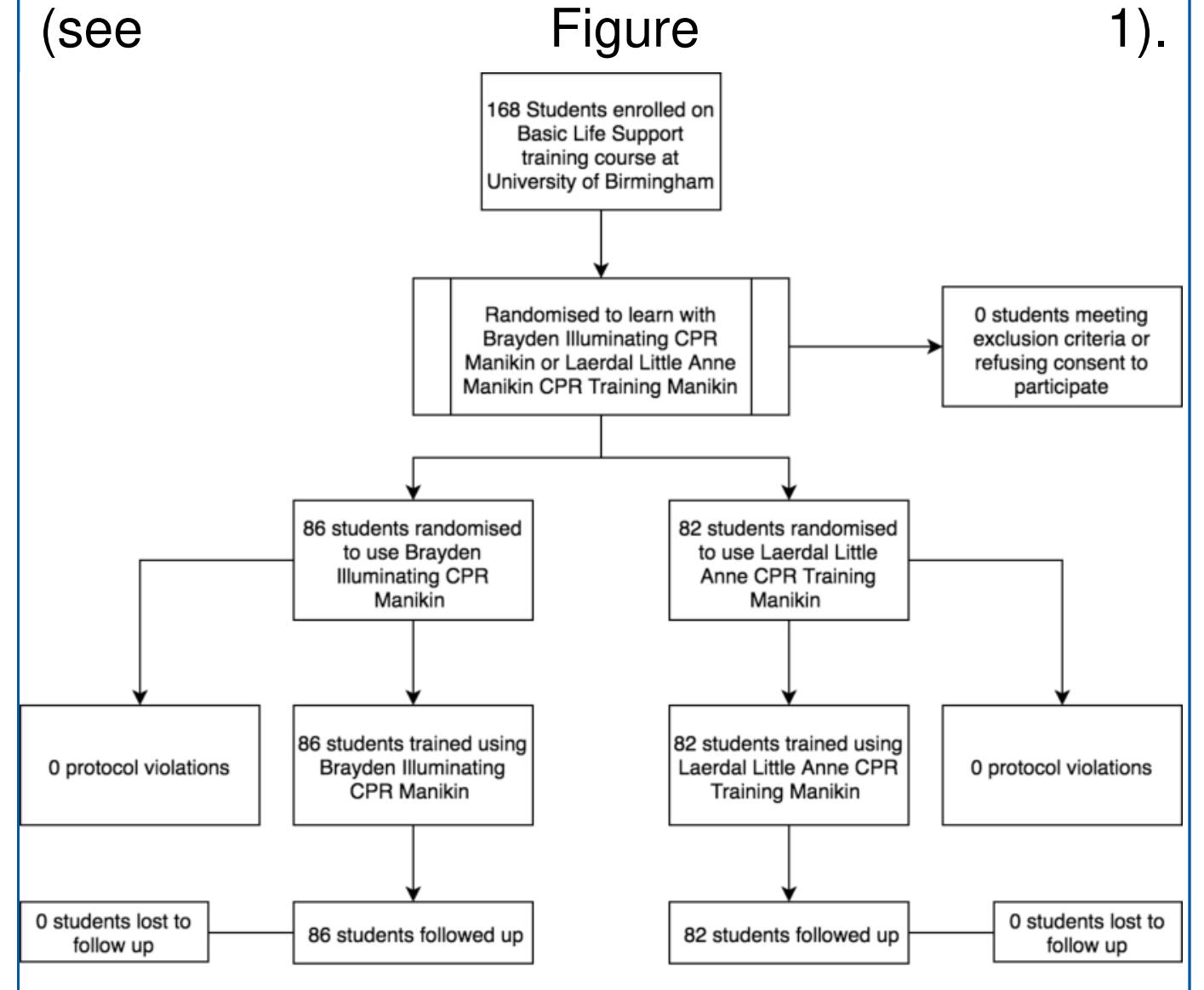

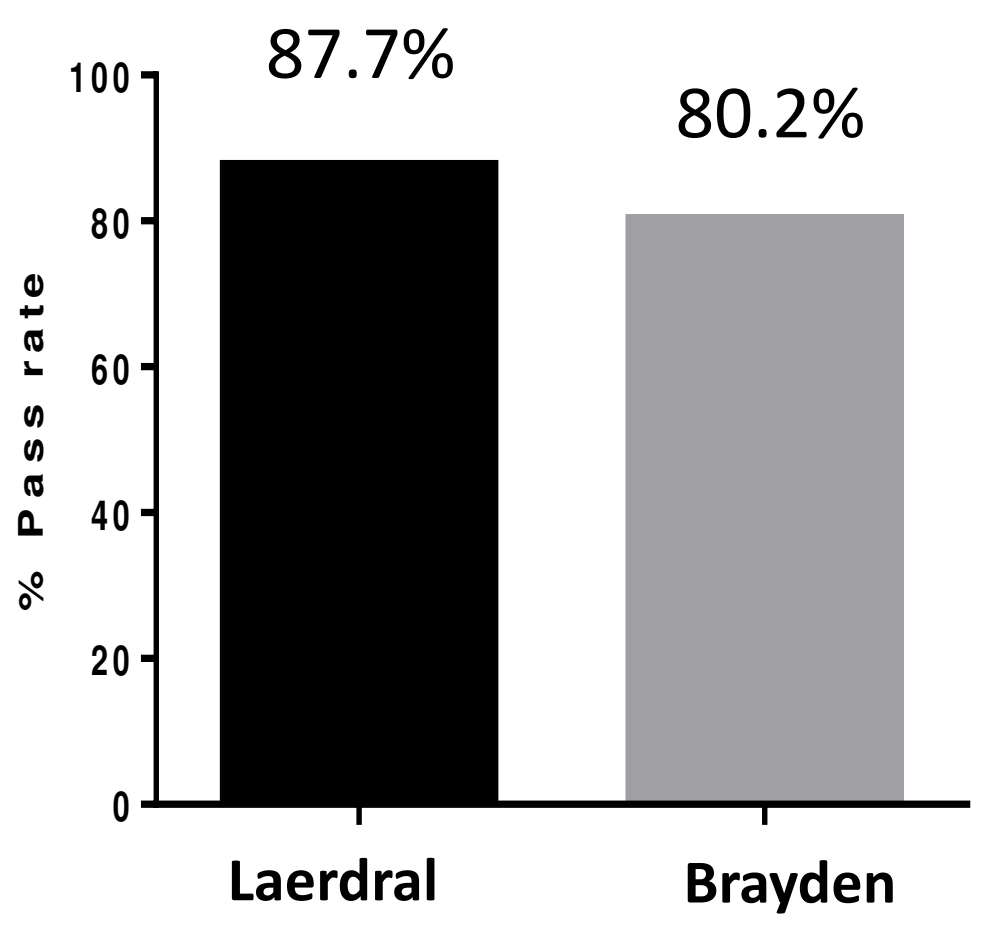

Figure 2. Pass rates were lower in the Brayden manikin group by $7.5 \%$.

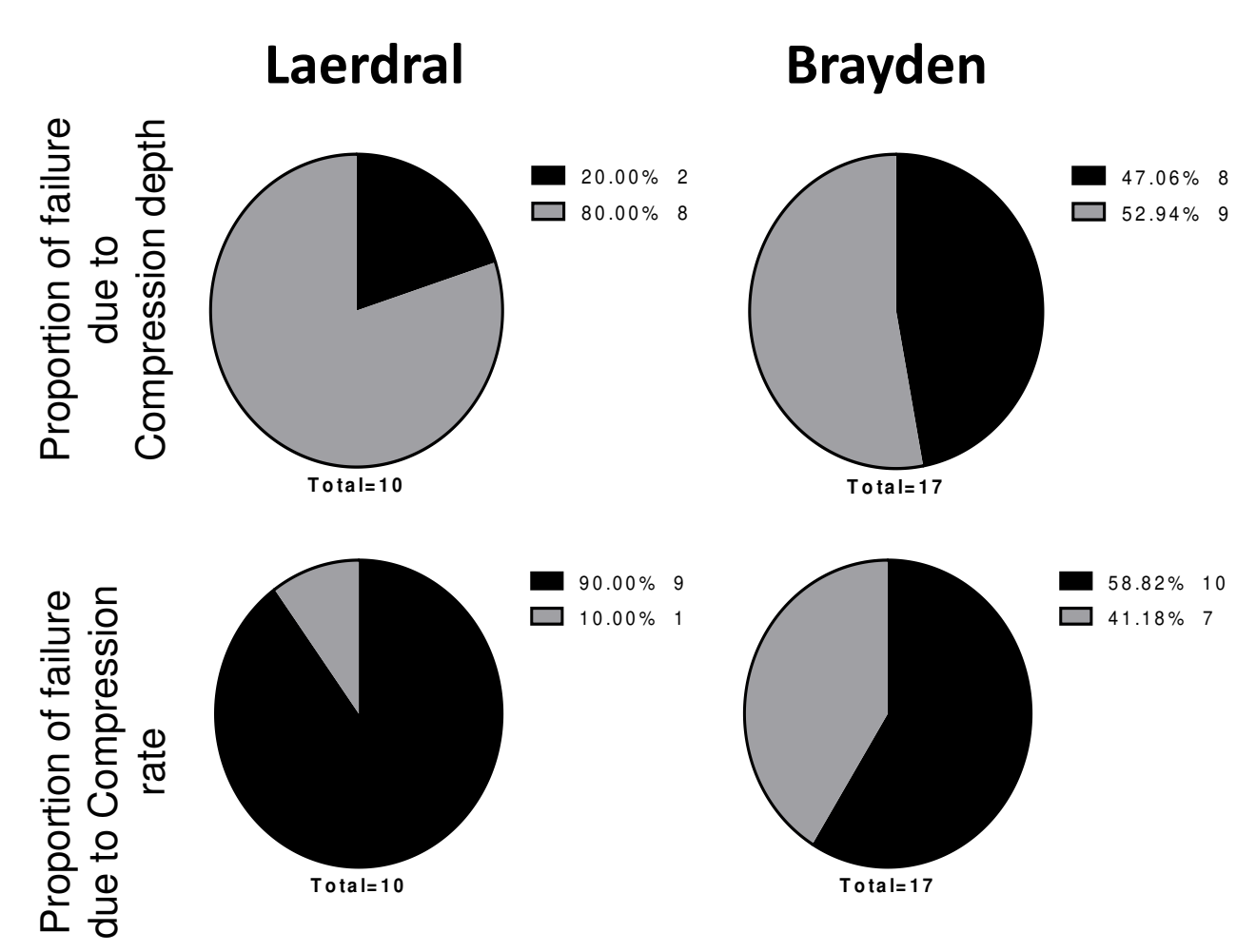

Figure 3. The Brayden manikin group had an improved compression rate, compared to that of the Laerdal group $(+31.18 \%)$, but this seemed to come at the expense of a poorer compression depth $(-27.06 \%)$.

Percent correctly released chest compressions

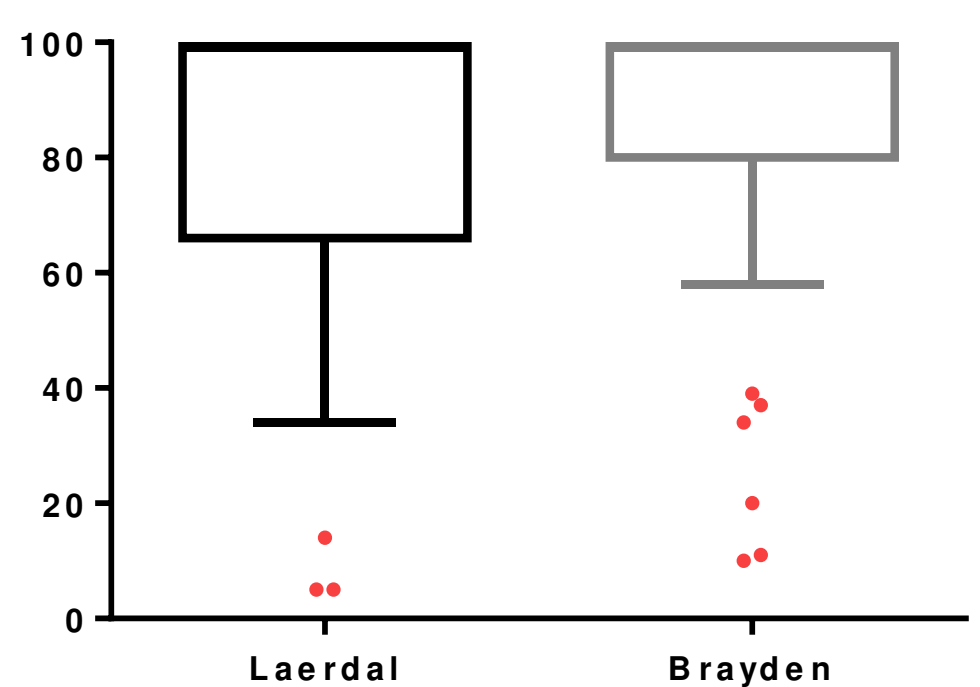

Figure 4. Percentage of correctly released compressions. While both groups had the same median (100), the mean percentage was $97.47 \%$ (SD 5.34) in the Brayden group (vs $90.56 \%$ (SD 17.14)).

Percent that reached adequate depth of chest compressions

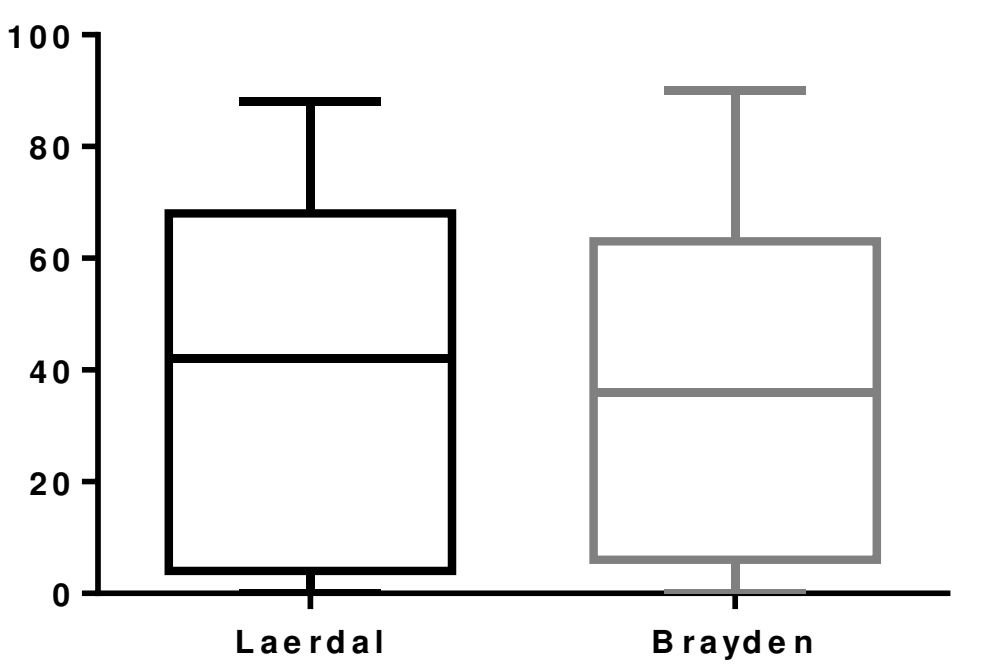

Figure 5. Percentage of candidates that reached adequate depth of chest compressions. The median was 42 in the Laerdal group and only 36 in the Brayden group. The mean percentage was lower in the Brayden group $(37.95 \%$, SD 28.58$)$ compared to that of the Laerdral group (41.39\% SD 28.58).
Percent of time spent at adequate chest compression rate

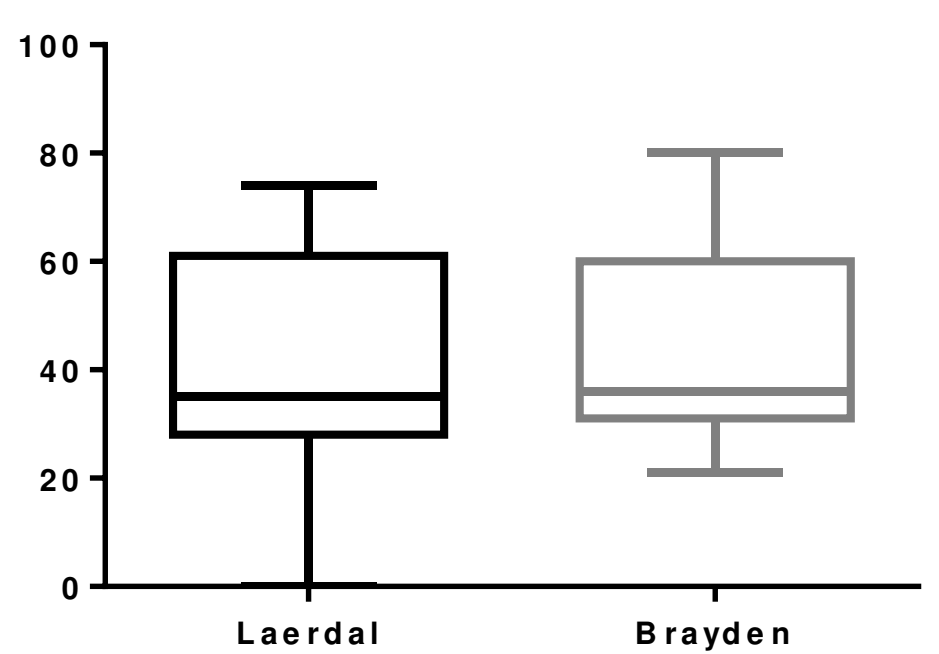

Figure 6. Percentage of time spent performing an adequate chest compression rate. The median was higher in the Brayden manikin group (36) compared to the Laerdal group (35). The mean percentage was $42.91 \%$ (SD 16.35) in the Brayden group (vs $41.55 \%$ (SD 19.18).

\section{Conclusion and discussion:}

Brayden manikins do not seem to increase exam performance in a trained, motivated cohort of BLS learners, and that improvements in compression rate might come at the cost of worse depth. Confusion about the illumination feedback is detailed in our other work, and may explain some of these effects. Further work is planned to investigate Brayden's use in a school age population, and in ad-hoc training where less time is available to students to perfect skills through repeated iteration.

\section{References:}

1. Hasselqvist-Ax I, Riva G, Herlitz J et al. Early Cardiopulmonary Resuscitation in Out-of-Hospital Cardiac Arrest. N Engl J Med

2015;372(24):2307-15.
2. Abella B, Alvarado J, Myklebust $\mathrm{H}$ et al. Quality of cardiopulmonary 2005;293(3):305-10 Initiativen to $\mathrm{M}$, Lippert FK, Folke F, et al. Association of National Initiatives to Improve Cardiac Arrest Management With Rates of Bystander Arrest. JAMA. 2013:310(13):1377-1384. doi:10.1001/jama.2013.278483 4. Harvey, P.R. et al. Peer-led training and assessment in basic life suppon for healthcare students: Synthesis of literature review and fifteen years practical experience. Resuscitation. 2012. 83(7):894 - 899 